\title{
Redefiniendo el precariado desde la perspectiva China $^{1}$
}

\section{Redefining the precariat from the Chinese perspective}

\author{
Borja, García Vázquez ${ }^{2}$
}

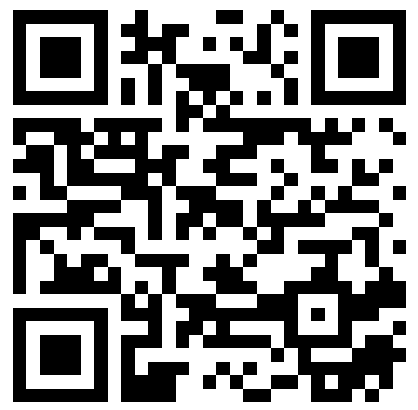

Fecha de recibido: 05-01-2021

Fecha de aceptado: 04-03-2021

\section{(cc) BY-NC-ND}

Esta obra está bajo una licencia de Creative Commons Reconocimiento-NoComercialSinObraDerivada 4.0 Internacional

\section{RESUMEN}

El presente artículo es producto de una revisión documental cuyo objetivo consistió en negar la originalidad del precariado como nueva clase social emergente, por medio de la exposición del concepto, confrontándolo a la noción de clase expuesta desde el marxismo, y las condiciones sociales existentes en la actualidad en República Popular China. Para ello se aplicó el método cualitativo de revisión documental de los trabajos del autor que acuñó el concepto de precariado, Guy Standing, oponiéndolo a escritos de Antonio Gramsci, Karl Marx, Louis Althusser, Mao Tse-Tung, y Slavoj Žižek, como representantes del marxismo; encontrándose que el empeoramiento de la situación de los trabajadores ha originado un fenómeno de precarización de sus condiciones de vida, concluyéndose que el precariado no es una nueva clase, sino la pauperización del proletariado.

Palabras claves: Clase trabajadora, crisis económica, ecologismo, marxismo, precariado.

\begin{abstract}
This article is the product of a documentary review whose objective was to deny the originality of the precariat as a new emerging social class, by exposing the concept, confronting it with the notion of class exposed from Marxism, and the existing social conditions in the currently in the People's Republic of China. For this, the qualitative method of documentary review of the works of the author who coined the concept of precariat, Guy Standing, was applied, opposing it to the writings of Antonio Gramsci, Karl Marx, Louis Althusser, Mao Tse-Tung, and Slavoj Žižek, as representatives of the Marxism; finding that the worsening of the workers' situation has originated a phenomenon of precarious living conditions, concluding that the precariat is not a new class, but rather the impoverishment of the proletariat.
\end{abstract}

Keywords: Economic crisis, environmentalism, Marxism, precariat, working class

Cómo referenciar este artículo:

García., V., B. (2021). Redefiniendo el precariado desde la perspectiva China. Revista Politica Globalidad y Ciudadanía, 7(14), 206-232. https://doi.org/10.29105/pgc7.14-10

\footnotetext{
${ }^{1}$ Artículo derivado del proyecto de investigación, la evolución del pensamiento socialdemócrata en Iberoamérica.

${ }^{2}$ Universidad Autónoma de Nuevo León, Monterrey, México. Doctor en Métodos Alternos de Solución de Conflictos, por la Universidad Autónoma de Nuevo León. Profesor de Derecho Internacional público en la Facultad de Derecho y Criminología de la Universidad Autónoma de Nuevo León. Email: borjagarcia131@gmail.com. Orcid: 0000-0003-0055-6917.
} 


\section{1.- INTRODUCCIÓN}

Guy Standing ha desarrollado en su trabajo académico el planteamiento del precariado, como una nueva clase social aún en vías de formación. Un supuesto que no merece tal posición si se contraponen sus fundamentos desde argumentos marxistas, y confrontando su hipótesis con las condiciones dadas en China (país heredero de esta tradición, hecho que motivó su elección como objeto de estudio al ser el principal Estado sucesor de esta escuela de pensamiento), desde las coordenadas del neoliberalismo, entendiendo como tal: un modelo doctrinal globalizante, destinado a lograr la construcción de un sistema organizativo, cuyo fin es garantizar la hegemonía de la democracia en lo político, el libre mercado en lo económico, y los Derechos Humanos en lo social.

Para ello, el neoliberalismo se sirve de la difusión y adhesión a estos pilares (con independencia de las contradicciones que puedan darse entre ellos), ya sea por procedimientos voluntarios (mediante el fomento ofrecido desde las distintas instituciones y fondos internacionales) o por la fuerza (en los procesos de reconstrucción, tras contiendas bélicas o luchas intestinas). Todo ello lleva al progresivo debilitamiento de las estructuras estatales/públicas en favor de operadores privados (tendentes al monopolio), y a la homogeneización y aculturación de las personas a escala planetaria, a efectos de delimitar su acción por sus hábitos de consumo.

Con el propósito de reformular la interpretación propuesta por Standing, se expone qué se entiende por precariado, el medio en que se desarrolla esta hipotética clase incipiente, cómo el neoliberalismo y la globalización han incidido en China, comprobando si pueden deducirse las condiciones del precariado en este país, explicando a continuación los argumentos que deben ser utilizados por la precarización de los trabajadores, como frentes de unión hacia la cuestión medioambiental y sanitaria, para concluir con las reflexiones finales.

\section{2.- FUNDAMENTO TEÓRICO}

\section{La clase social en Marx}

Guy Standing, artífice del neologismo precariado (como fusión del adjetivo precario y el nombre proletario) alude bajo esta denominación a una doble conceptualización, como grupo socio-económico, 
García., V., B.

justificando su empleo ante las nuevas relaciones de clase motivadas por la economía de mercado (Standing, 2011: 7).

Debe indicarse que por clase social se comprende a:

"grandes grupos de personas que se diferencian unas de otras por el lugar que ocupan en un sistema de producción social históricamente determinado, por su relación (en la mayoría de los casos fijada y formulada en la ley) con los medios de producción, por su papel en la organización social del trabajo y, en consecuencia, por la magnitud de la parte de riqueza social de que disponen y el modo en que la obtienen" (Harnecker, 2020: 222-223).

Marx no llegó a ofrecer una definición exacta del concepto de clase, dejando inconcluso con su muerte el capítulo destinado a resolver dicha incógnita, el cual ocupa el último apartado del volumen III de su obra culmen, El Capital. Su máxima descripción consistió en diferenciar grupos humanos conforme a la posición que ocupaban en el modo capitalista de producción, de acuerdo con la propiedad y forma de obtención de concretos réditos: asalariados (propietarios de fuerza de trabajo que obtienen un salario por su trabajo); capitalistas (propietarios de capital, que sustraen una ganancia de aquel); y terratenientes (propietarios de la tierra, que sustraen de ella una renta) (Marx, 2009:1123).

Los asalariados, también denominados proletariado, supondrían el escalón inferior dentro del esquema de clases en las relaciones de producción. Al margen de esta división, se encontraría por debajo del proletariado el lumpenproletario, un estrato carente de conciencia de clase. Empleado para explicar históricamente la posición intermedia entre el hombre libre romano (plebeyo) y el esclavo (Marx, 1846), en su contexto social el lumpen representaba el "producto pasivo de la putrefacción de las capas más bajas” (Marx, 2018: 136), “una masa bien deslindada del proletariado industrial (...) rateros y delincuentes de todas clases, que viven de los despojos de la sociedad, gentes sin profesión fija, vagabundos, gens sans feu et sans aveu” (Marx, 1850), conformado por:

\footnotetext{
“arruinados, con equívocos medios de vida y de equívoca procedencia, junto a vástagos degenerados y aventureros de la burguesía, vagabundos, licenciados de tropa, licenciados de presidio, huidos de galeras, timadores, saltimbanquis, lazzaroni, carteristas y rateros, jugadores, alcahuetes, dueños de burdeles, mozos de cuerda, escritorzuelos, organilleros, traperos, afiladores, caldereros, mendigos, en una palabra, toda es masa informe, difusa y
} 
errante que los franceses llaman la bohème (...) hez, desecho y escoria de todas las clases" (Marx, 1852).

Teniendo en cuenta los principios de que partía Marx para identificar una clase, conforme a su posición y conciencia de la misma, llevaron a Harnecker (2020) a exponer que:

"Las clases sociales son grupos sociales antagónicos, en que uno se apropia del trabajo del otro a causa del lugar diferente que ocupan en la estructura económica de un modo de producción determinado, lugar que está determinado fundamentalmente por la forma específica en que se relaciona con los medios de producción” (222-223).

\section{El precariado y su mundo}

El precariado (embrión de una supuesta nueva clase social) conformado por aproximadamente una cuarta parte de la población adulta (Standing, 2011:24), sin que existan intereses comunes ni unidad material entre sus miembros (Standing, 2014a:12), se caracteriza por experimentar cuatro estados anímicos: ira, por el malogramiento de las opciones de movilidad social ascendente; anomia, causada por la impotencia de cambio de la realidad por sus integrantes; ansiedad, respondiendo a las circunstancias de continua inseguridad socio-laboral; y finalmente alienación, no en el sentido clásico del proletariado, de cumplimiento de las funciones para un propósito ajeno, sino adicionando un sentimiento de engaño, fruto de la constante exposición a la exhortación del pensamiento positivo por su trabajo realizado (Standing, 2011:19-21), el conocido mindfulness, empleado en la reducción de estrés para tratar la depresión en adolescentes y adultos jóvenes, con efectos moderados (Chi, Ai, Tingting, Peichao, Chi, 2018).

Desde los parámetros descritos por Standing pueden distinguirse tres grupos, como componentes de esta supuesta nueva clase social en formación, que impiden su constitución como clase por sí misma:

Primero, los excluidos del proletariado, hijos de trabajadores, con escasa formación educativa y privados de los servicios a que tenían acceso sus ascendientes (Standing, 2014b:11), convirtiéndose en el caldo de cultivo idóneo al populismo nacionalista (Standing, 2014a:13), es decir, los herederos repudiados por el Estado de bienestar postfordista, que ven amenazadas sus posibilidades de prosperidad social en los dirigentes políticos y en el exterior de sus países;

Segundo, las minorías étnicas y los inmigrantes (denominados por el autor como nostálgicos, aunque nos oponemos a este apelativo en la medida que comparten ese sentimiento con los hijos de los 
García., V., B.

antiguos proletarios), poco visibles en el apartado político (Standing, 2014a:14), deducimos por su falta de integración, en la cual cabrían incorporar los descendientes de esos expatriados, que añoran un mundo idílico que nunca conocieron, y al que se aferran para construir sus relatos de oposición al sistema (como se ha visto con el fenómeno del yihadismo en Europa);

Por último, una generación de profesionales surgidos de la academia, con alta cualificación, y con preparación suficiente para lograr prosperar frente a las frustraciones y privaciones a que son sometidos (Standing, 2014 $4^{\mathrm{a}}: 13-14$ ), con aspiraciones a mejorar la sociedad sobre los valores progresistas de la igualdad, la libertad y la sostenibilidad ecológica (Standing, 2014b:11), con opciones de convertirse en los portadores de la antorcha de la tradicional intelligentsia.

A ellos podrían sumarse: los jóvenes frustrados en sus expectativas de vida (con tasas de desempleo superiores en hombres que en mujeres desde la crisis de 2008); adultos mayores que son despedidos de sus empleos a causa de la edad, viéndose forzados a encontrar trabajos temporales para no caer en la pobreza; demandantes de asistencia sociales del Estado; personas con necesidades especiales que no reciben dichas ayudas; y la población reclusa (que en países como China e India son empleados como mano de obra barata) (Standing, 2012: 596-597).

En estas personas se aprecia que sus relaciones de trabajo se basan en situaciones laborales fluctuantes, convertidas en una constante nebulosa de incertidumbre temporal y volatilidad física del empleo, sin estar amparadas por condiciones garantes de las necesidades de reproducción, generando trabajo forzado y alienamiento a los que no puede oponerse el individuo (en un persistente desapoderamiento sobre sus aptitudes y gestión del tiempo material); originando una imposibilidad de satisfacer las expectativas de vida de las personas que se encuentran en esta situación de sometimiento, pese a disponer de una especialización educativa superior a las posibilidades de su hipotética acción productiva, aunque necesaria, en tanto opera como filtro de acceso a las opciones de empleo vacantes (Standing, 2014a $: 8$ ), en sintonía con la finalidad de conseguir trabajadores genéricos o “todo terreno”, que puedan ser desechados, sin conllevar dificultades a sus patronos (Bauman, 2012:22).

Asimismo, la vida del precariado discurre en la búsqueda de obtención de trabajo, al cual debe destinar medios y tiempo equivalentes a los de una actividad laboral, tal y como formula Standing en la máxima “trabajo para buscar trabajo”, quedando excluido del mercado formal, y por extensión, del acceso a la cobertura adicional ofrecida desde las diversas regulaciones (Standing, 2014a:9). Uniéndose 
a este grupo otras características como son la negación de derechos culturales, sociales y políticos, por carecer de residencia legal a causa de la concatenación de empleos flotantes (Standing, 2012: 591).

El escenario en que transcurre el precariado, se delimita entre las coordenadas del agotamiento de la ilustración y sus propuestas quiméricas (Contreras Natera, 2015: 132). Un tiempo en el que la mutabilidad de las circunstancias impide la cristalización de nuevos elementos de guía para la toma de decisiones sociales, impidiendo planteamientos más allá del corto plazo; con la quiebra del poder del Estado-nación, frente al avance de fuerzas transnacionales y la incapacidad de las instituciones para intervenir a nivel global, llevando a un paulatino abandono de la acción interna de atención de su población, en favor de la acción externa (encuadrada en la competitividad internacional), que apareja un creciente proceso de vaciamiento de los organismos (a través de la externalización de servicios) y descrédito institucional, por el desamparo e inoperatividad que ofrece (Bauman, 2008:7-9); a la par, que aumenta la uniformidad global, en el proceso generador de desarraigo común "en nombre del desarrollo y del progreso” (Contreras Natera, 2015: 129).

El precariado no es contrario a la política, pero se encuentra desconectado de ella por cuanto esta no es capaz de entender sus aspiraciones y necesidades (Standing, 2014b:12), lo que explica el crecimiento de la desafección ciudadana hacia los medios representativos tradicionales, y la procrastinación ante un mundo irreal, de aparente libertad ofrecida por el mercado, con infinitas posibilidades de consumo, pero solo para aquellos que gozan del Capital para intervenir en él; constituyendo una ficción alimentada por los aparatos ideológicos de esta sociedad consumista de productos, que no están al alcance del precariado, al no disponer ni de la capacidad de reproducir sus propias condiciones de producción, siendo así personas desechables por el sistema, en un retorno a la servidumbre.

Los aspectos para alcanzar la consolidación de esta clase, son identificados por Standing en el reconocimiento público, la obtención de representación institucional, y una redistribución de los medios existentes para materializar las condiciones personales y laborales que permitan una sociedad sustentable, a través de la seguridad económica, el control sobre el tiempo, espacios de calidad, educación liberadora, conocimiento financiero y Capital (Standing, 2014b:12); pero en suma, todo ello no es sino un error, en la medida en que tales fines no conforman la identificación de las personas en precario como una nueva clase social, sino las condiciones para lograr que los parias laborales puedan ser reintegrados y autoidentificados en la clase trabajadora. 
García., V., B.

\section{Neoliberalismo y globalización}

Althusser defendía dos tesis conjuntamente "1. No hay práctica sino por y bajo una ideología. 2. No hay ideología sino por el sujeto y para los sujetos” (Althusser, 2018:59). En el marco de la globalización, entendida tanto en el ámbito de interacción alcanzado por los países tras la disolución de la Unión Soviética (URSS), como por el desarrollo de las comunicaciones, que ha incrementado entre todos los Estados la interrelación en el plano ambiental y económico mundial (Kwan Chan, Peng, 2011:423), se ha consolidado una ideología hegemónica, el neoliberalismo.

Marx ya explicaba como "mientras la burguesía de cada nación seguía manteniendo sus intereses nacionales aparte, la gran industria creaba una clase que en todas las naciones se movía por el mismo interés y en la que quedaba ya destruida toda nacionalidad” (Marx, 2017:93). Un grupo sin identificar, cosmopolita, trascendente a las fronteras, que no conoce más límites que los que le impone su Capital, y que es capaz de configurar las relaciones descendientes hacia su clase.

El neoliberalismo ha tratado de formar un sistema cuyos postulados consisten en garantizar la libertad del individuo a su más alta expresión, mercados sin intervención gubernamental ni políticas discrecionales, así como reformas estructurales que permitan la máxima desregulación de los mismos (Hirai, 2015:9), hecho que ha demostrado como el mercado global daña a los débiles, y solo asegura movilidad a los altamente cualificados (Birdsall, 2006:433) o posicionados socialmente; aunque desde el plano formativo, las instituciones de educación superior mantienen vínculos de dependencia financiera y legitimadora de los Estados en cuyo territorio actúan, por ser mayormente pertenecientes al sector público (Marginson, Van Der Wende, 2009: 25).

Igualmente, en el plano internacional, no es probable el desarrollo de un modelo análogo a la regulación y fiscalización de los mercados por los gobiernos nacionales (que ayudaría a conseguir la igualdad de oportunidades) (Birdsall, 2006:434); por lo que el componente formativo y de alta movilidad laboral, queda determinado por el factor nacional, y su restricción solo es evadida por aquellos pertenecientes a la clase hegemónica (los estertores de la élite industrial, y sus sucesores en este nuevo periodo postindustrial), perpetuándose en el marco de la globalización las rivalidades entre los trabajadores de distintas nacionalidades.

El 25 de diciembre de 1991, fecha de desaparición de la URSS, constituye la derrota histórica del primer y único intento de creación de un modelo global y alternativo al capitalismo, conllevando el 
retraimiento (cuando no la disolución) de pensamientos divergentes al neoliberalismo; el cual se alzó como sistema victorioso, sin más oposición ni límites que los de su propia capacidad de producción, y sin que su heredero ideológico, la República Popular China, haya ofrecido un sostén de pensamiento (y carezca de intención de hacerlo). Los Estados Unidos, en su convencimiento de superioridad de ideario, predicando su victoria de la guerra fría, ejercieron una política de libre comercio con China, en la certeza de que el crecimiento que llevaría aparejado, traería consigo una mejora de sus instituciones y la democracia al país (cosa que no ha sucedido) (Acemoglu, Robinson, 2012: 517).

China, como apunta Žižek:

“constituye hoy en día el Estado capitalista ideal: libertad para el capital, con el Estado llevando a cabo el trabajo sucio de controlar a los trabajadores", en lo que resulta ser "una nueva clase de capitalismo: indiferencia hacia las consecuencias ecológicas, represión de los derechos laborales, todo subordinado al implacable impulso al desarrollo y a la conversión en la nueva superpotencia” (Žižek, 2013a:30).

Su expansión económica es evidente, siendo el mayor exportador y tenedor de divisas del mundo (Ohashi, 2015:234), al ser en 2018 más del 15\% del Producto Interior Bruto mundial (The World Bank, 2021a), y con el 18,34\% de la población del planeta, sus hogares tienen un crecimiento anual de consumo de un 9,5\% (The World Bank, 2021b), en un contexto de globalización, un proceso geoespacial de mayor convergencia e interdependencia de las esferas de acción mundiales y/o regionales (Marginson, Van Der Wende, 2009:19). Esto causa un aumento de la conectividad multinivel, donde no solo las empresas, sino especialmente los individuos, asisten a un constante flujo de (des)información, reduciendo las sensaciones de distanciamiento geográfico (aunque sean ficticias).

El problema en torno a la globalización reside en quién dirige la economía global y fija sus intereses, resaltando la importancia que representa el elemento político y de poder (Birdsall, 2006:429) en la respuesta a esta controversia. Una problemática en la que se encuentra China, por su expansión comercial y el aumento de su presencia en todos los mercados.

Desde que en 1978 Deng Xiaoping puso fin a las políticas aislacionistas (época en que el país representaba el $1 \%$ del comercio mundial) permitió entre otras cuestiones, las inversiones extranjeras, que se han incrementado exponencialmente en estas décadas a través del descenso arancelario, desde el 45\% que fijaban en 1992, a menos del 7\% en 2001, fecha la que el país ingresó en la Organización 
García., V., B.

Mundial del Comercio (logrando aumentar su presencia internacional), y en 2013 representar el $11 \%$ del comercio mundial (Irwin, 2015:202) consiguiendo que más de 500 millones de personas escapasen de la pobreza (Ohashi, 2015:239); pero con un aumento de la desigualdad, como constata la evolución del índice de Gini, del 32,2 en 1990 al 43,7 en 2010 (Banco Mundial, 2021). Todo ello sin olvidar la represión sistemática, cuyo punto álgido se alcanzó en el contexto de las manifestaciones de la plaza de Tiananmen en 1989, con alrededor de 5.000 heridos y 1.500 muertos (Tamames, Debasa, 2013), y más recientemente en las protestas de Hong Kong de 2019, que sin alcanzar las cotas del pasado siguen mostrando las demandas de mayor libertad de la población china.

El país se ha organizado históricamente en torno al danwei, un término empleado para designar la integración espacial, del trabajo, la residencia y la vida social de la ciudadanía, efectuada por el Partido Comunista chino (Bjorklund, 1986:21), que sigue siendo la principal unidad de organización territorial y laboral de la población urbana (Xie, Wu, 2008:561-562), y que en opinión de Standing (2012: 594) al ser subvencionado por China, ha motivado el incremento de la oferta laboral mundial, el aumento en la desigualdad de ingresos, y una pérdida en la capacidad de negociación de los trabajadores de todo el mundo.

Las reformas de Deng Xiaoping, conocidas como "las cuatro reformas" y "la apertura de puertas”, implementadas desde finales de la década de 1970, supuso el desmantelamiento de las políticas soviéticas y maoístas, y el comienzo de la economía de mercado en China (Ng, Warner, 2002:10), estableciendo el fin del conocido "tazón de arroz de hierro”, expresión con que se aludía al sistema garantizado por el Estado, de alimentación, pensiones, vivienda, atención sanitaria, educación, transporte y ocio, para los trabajadores y sus familias (Hughes, 1998:71). En este escenario habría que entender el precariado como la progenie no reconocida del neoliberalismo, fruto de un constante abaratamiento de la vida humana, que les dirige al desamparo, incumpliendo las reglas básicas de reproducción, pero sin que constituya una nueva clase, tratándose exclusivamente de trabajadores excluidos de las necesarias garantías sociales.

En palabras de Marx, "El nivel mínimo de salario, y el único necesario, es lo requerido para mantener al obrero durante el trabajo. Y para que él pueda alimentar una familia y no se extinga la raza de los obreros" (Marx, 1844); pero el precariado es la devaluación del conjunto de mínimos vitales hasta el máximo posible (antes de producirse su supresión), cuya razón de ser responde al enfrentamiento internacional, entendible desde la dialéctica de Estados. 
Esta afirmación es comprendida desde la exposición de Xin Meng, al sostener que:

"sí China quiere convertirse en una superpotencia económica, su estructura industrial debe estar dominada por una industria de capital intensivo, de alta tecnología" de forma que "el crecimiento económico futuro se base en la innovación y la sofisticación tecnológica, y no en la mano de obra barata", dejando de ser "la fábrica del mundo", para convertirse en "el laboratorio del mundo" (Meng, 2014:380).

Irónicamente, el país ya ha sido objeto de uno de los mayores experimentos sociales de la historia (si excluimos el sistema de casta que durante siglos rigió la India) a través de la política del hijo único. Esta campaña, que será recordada “como una de las lecciones más costosas de la formulación errónea de políticas públicas” (Feng, Gu, Cai, 2016:84), fue establecida en 1980 con la finalidad de reducir las altas tasas de natalidad del país y controlar el crecimiento demográfico, con el propósito de que no interfiriese en el desarrollo económico del país (Neuman, Schmitz, 2018); constatándose, durante el periodo que estuvo en vigor esta política, un descenso de nacimientos por cada 1.000 personas, de 33,4 que se registraban en 1979, a 4,95 en 2012 (Song, 2014:113), causando efectos en la sociedad.

No obstante, a que el experimento finalizó en 2015, perdura la mentalidad de los padres a focalizar sus esfuerzos en tener solo un descendiente, para "que pueda ascender en la escala social o que al menos no decaiga en ella" (Fifield, 2019). Igualmente debe destacarse la realidad de los shidu, término chino que alude a los padres que han perdido a su único hijo, estimándose en 2010 que se encontraban en esta situación 2.412.600 familias (Song, 2014:113).

Dichas personas deberán afrontar una vejez en soledad conviviendo con el sentimiento de pérdida, que les conduce al aislamiento y al progresivo deterioro de sus facultades mentales, detectándose frecuentemente la aparición de patrones autistas (Li, 2013: 551), sin omitir la discriminación laboral a la que se enfrentan los individuos con mayor edad, agudizado en China, que cuenta con una edad oficial de jubilación a partir de los 45 años en las mujeres, y de los 55 en el caso de los hombres, edad que es adelantada en las familias shidu (Song, 2014:114).

En 2013 la nación tenía 770 millones de trabajadores, de los cuales el 72\% provenía del ámbito rural (Meng, 2014:383), con un desempleo del 4,6\%, idéntico al que mantenía en 2003, y superior al 2,7\% que registraba en 1993 (The World Bank, 2021c) de una población total de 1.178 millones de 
García., V., B.

habitantes, frente a los 1.357 millones de 2013 (The World Bank, 2021d), lo que equivale a 62 millones de personas sin empleo en China.

Además el país cuenta con una migración interna, que en 2014 era de 270 millones de desplazados del campo a la ciudad (Zhao, 2019), de los cuales un 60\% se desempeña en trabajos precarios, desarrollando sus actividades en el plano de la economía informal, sin estar amparados por las regulaciones laborales, desempeñándose en sectores como la construcción, los servicios, el trabajo doméstico, cuidado de menores y el reciclaje (Swider, 2015:5), reflejándose la situación de millones de personas que migran aspirando a obtener un futuro.

Se trata de los nacidos a partir de 1980, una generación rupturista con las condiciones de vida de sus padres, con educación secundaria en el 67,2\% de sus integrantes, pertenecientes a familias pequeñas como consecuencia de la política del hijo único, a quienes no agradan el estado del empleo en las fábricas, junto a los bajos salarios, en comparación con el aumento de los costes de vida (Béja, 2011:3), generando malestar en la población, tal como acreditó en 2008 la universidad de Fudan, al realizar una encuesta a 30.000 migrantes de las principales ciudades del país, donde se obtuvo que solo el 7,6\% estaba satisfecho con su vida en la ciudad (Milcent, 2010:35).

Del mismo modo, no puede obviarse a los ciudadanos que se han ido del país en una diáspora, que en la década de 1980 era superior a 25 millones de personas, y a comienzos del siglo XXI, rondaba los 40 millones (Fleischer, 2012:72), llevando a algunos de sus integrantes, hijos de inmigrantes que no hablan el idioma chino al no haberlo aprendido, a reflexionar sobre lo "precaria, inestable y problemática formación de su identidad, su complejidad social y las repercusiones culturales” (Ang, 2013:18); y en otros casos siendo criticada la falta de profundidad de análisis, al tratar a la población china como un único grupo étnico, en detrimento de las minorías que lo conforman (como tibetanos e uigures) con fines de explotación política y de clase (Lo, 2013:173).

La visión defendida por Antonio Gramsci, de implementación del utilitarismo en el mundo laboral, para conseguir el máximo rendimiento productivo a través de la organización de la vida individual y colectiva, permitiendo resolver las contradicciones "que no pueden dejar de existir", a partir de la creación de "un nuevo conformismo desde abajo", idea que asociaba a la autodisciplina como libertad individual (Gramsci, 2009:226-227), puede entenderse como el logro condicionado de la libertad del sujeto, en tanto este cumpla con el cometido que el sistema espera de sí mismo. Estas ideas parecen 
haber sido recogidas por el neoliberalismo, pero en una vuelta del revés perversa que no espera del sujeto un sacrificio consciente en favor de la comunidad, sino inmolarse silenciosamente. Sirva de ejemplo que entre enero y agosto de 2010, en la fábrica Foxconn de Shenzhen donde se fabricaban teléfonos iPhone, se suicidaron 17 trabajadores cuyo salario en las líneas de montaje era de 1.000 yuanes al mes (Béja, 2011:3) (aproximadamente 140 dólares).

Debido a la presión a que se ven sometidos los individuos en el país asiático, han proliferado las protestas laborales desde las 87.000 convocadas en 2005 (Lansdowne \& Wu , 2009: 10), a 500 movilizaciones diarias (O’Brien \& Deng, 2017: 180), lo que supone más de 182.000 protestas al año. De ellas destaca lo ocurrido en 2014 en la fábrica textil de Yue Yuen, donde aconteció la mayor huelga de la historia de China, tras hacerse público el impago del seguro social a los trabajadores (Estlund, 2017: 13) muchos de los cuales se desempeñaban en la empresa en condiciones de temporalidad por razón del seguro (Tapia, Elfström, \& Roca-Servat, 2018: 194).

Ya Marx defendía como "La demanda de hombres regula necesariamente la producción de hombres, como ocurre con cualquier otra mercancía. Si la oferta es mucho mayor que la demanda, una parte de los obreros se hunde en la mendicidad o muere por inanición” (Marx, 1844).

Esta es la realidad a que se asiste en buena parte del mundo, y es la causa del aumento del precariado, la progresiva pérdida de oportunidades y el fracaso de expectativas (laborales y sociales), hasta sumir al individuo en una muerte en vida (cuando no a su supresión real), por la reducción de la humanidad a una concatenación de meros ajustes contables, de acuerdo a las conveniencias del sistema. Idea que era defendida por Gramsci al explicar la tarea educativa del Estado, como elemento capaz de adaptar los lineamientos morales de la masa popular a los requerimientos económicos de la producción, surgiendo así "los nuevos tipos de humanidad” (Gramsci, 1984:100); lo que explica la aparición del precariado no como una nueva clase, sino como la involución del proletariado (de acuerdo a las exigencias del sistema), a un estado de esclavitud invisible en el que el trabajador vive en un mundo panóptico.

Una ruptura con el hombre-masa existente desde antes de la revolución industrial, y que en la actividad fabril surgía "sobre la base de la posición ocupada por la colectividad en el mundo de la producción”, correlativa a la existencia del “hombre representativo”, cuya función de liderazgo ha ido retrocediendo (Gramsci, 2009:225), hasta extinguirse en el estado del precariado. Una actividad, la del intelectual, que ha sido clave en el desarrollo de cualquier movimiento, pues como reconocía Mao Tse- 
García., V., B.

Tung en 1957, "la gigantesca y ardua causa de la construcción socialista de China requiere que la mayor cantidad posible de intelectuales se ponga a su servicio", en un periodo en que se había “debilitado la labor ideológica y política entre los intelectuales y jóvenes estudiantes" (Tse-Tung, 2013:214), equivalente a la experimentada por el precariado, un proletariado desmemoriado tras haber sido deglutido y fagocitado por el sistema ideológico burgués, que ha neutralizado cualquier identidad de clase.

Gramsci exponía como la burguesía es “capaz de absorber a toda la sociedad, asimilándola a su nivel cultural y económico”, llevándole a declarar que siendo así “hasta el punto de concebir el fin del Estado y del derecho por haber llegado a ser inútiles y haber agotado su tarea y haber sido absorbidos por la sociedad civil” (Gramsci, 2009:211).

Por su parte Žižek define la postura de la clase media como un antagonismo puro, en la medida en que se autopercibe desde unas coordenadas de trabajo y moral, opuestas a "las grandes corporaciones, sin patria ni raíces, de un lado, y los excluidos y empobrecidos inmigrantes y habitantes de los guetos, por otro”, pero que enmascara el engaño de su supuesta imparcialidad social, desdibujando las líneas de clase (Žižek, 2008:22-23); hechos que explican la existencia del precariado, trabajadores sin garantías de protección ni unidad, cuyas mermadas condiciones materiales les recluyen en el conformismo y el individualismo, por causa de la acción constante del neoliberalismo y de la flaqueza de la intelectualidad y las fuerzas representativas. Una situación expuesta por Marx al decir que "la competencia aísla a los individuos, no sólo a los burgueses, sino aún más a los proletarios, enfrentándolos los unos con los otros, a pesar de que los aglutine” (Marx, 2017:94), lo que aclara la falta de disposición del precariado.

La revolución que puede llegar: la cuestión medioambiental y sanitaria como elementos catalizadores de la lucha:

"Los viejos dirigentes intelectuales y morales de la sociedad sienten que se les hunde el terreno bajo los pies, se dan cuenta que sus prédicas se han convertido (...) en algo ajeno a la realidad, en pura forma sin contenido (...) de aquí su desesperación y sus tendencias reaccionarias y conservadoras (...) piden al Estado que adopte medidas represivas, y se constituyen en grupo de resistencia apartado del proceso histórico real, aumentando de este modo la duración de la crisis, porque el ocaso de un modo de vivir y de pensar no puede producirse sin crisis. Por otro lado, los representantes del nuevo orden en gestación, 
por odio racionalístico contra lo viejo, difunden utopías y planes descabellados" (Gramsci, 2009:226).

En palabras de Standing "El precariado es una nueva clase social peligrosa en parte porque rechaza todas las viejas ideologías políticas predominantes y porque es intuitivamente transformador" (Standing, 2014a:15), pero cabría negar esta afirmación en tanto que el cambio no es concebible desde los parámetros de la desunión, y sin proyecto de futuro que pueda contraponerse a las circunstancias que se quieren modificar, como defendió (y demostró) Lenin desde su exposición en ¿Qué hacer? Al decir: "Sin teoría revolucionaria tampoco puede haber movimiento revolucionario".

La educación marxista es parte del currículo académico de los estudiantes escolares y universitarios del país, a fin de obtener personas cualificadas para la construcción del socialismo con características chinas (Australian Marxist Review, 2014), y la formación de líderes para el PCCh (Taber, 2018), logrando así el fomento de aplicaciones prácticas a dicha educación, como manifiesta su ministro en esta área (Qiuyan, 2017). La promoción gubernamental del acervo ideológico por medio de la enseñanza regulada, ha generado una contradicción interna propiciada por los propios estudiantes marxistas. Este hecho ha quedado visiblemente patente, por el arresto de aquellos universitarios que han fomentado huelgas y protestas en favor de las condiciones de trabajo de sus compatriotas (Yang, 2019).

La ideología no es sino la representación de la relación presente de las personas con sus condiciones de existencia materiales (Althusser, 2018:52-53), y sin ideología, lo único que encontramos es un individuo al que le han despojado de su humanidad. En opinión de Althusser "los hombres que no tiene el conocimiento de las realidades políticas, económicas y sociales en las que deben cumplir las tareas que les asigna la división del trabajo, no pueden vivir sin guiarse por una cierta representación de su mundo y sus relaciones con él” (Althusser, 2018:120). Por lo que necesariamente el rechazo de las viejas ideologías a las que alude Standing, debería dar lugar a la cristalización de una genuina ideología originada en las especiales condiciones materiales de esta clase emergente. Cosa que no es concluyente por la propia divergencia de sus miembros, y tampoco se demuestra de acuerdo a lo ocurrido en China, donde se produce una contradicción entre el estudio y ánimo de puesta en práctica de la ideología por el gobierno, y el acallamiento de sus practicantes por las autoridades.

\section{Como defiende Žižek:}


García., V., B.

"mientras persista esta esencial despolitización de la esfera económica" y "cualquier discurso sobre la participación activa de los ciudadanos" quede "reducido a una cuestión cultural en torno a diferencias religiosas, sexuales, étnicas o de estilos de vida alternativos (...) no se podrá incidir en las decisiones de largo alcance que nos afectan a todos” (Žižek, 2008:110).

Así, incumplida la máxima de Marx por la cual "Los diferentes individuos sólo forman una clase en cuanto se ven obligados a sostener una lucha común contra otra clase" (Marx, 2017:95), no puede concebirse el precariado como una clase original, sino los trabajadores pauperizados y desempleados como consecuencia de la liberación de los mercados y su globalización, que lleva a una competición aislacionista entre los individuos, y deshumanizadora entre los países, por ver quien consigue ofrecer más por menos. Una condición explicada por Standing (2008: 29), surgida de la flexibilización del mercado laboral en el plano de la generación de competitividad nacional, que provoca una facilitación de los despidos y la disminución de incentivos a la contratación, favoreciendo la generación de trabajo informal.

Existe una relación "entre la aparición de movimientos sociales con las insuficiencias del orden institucional existente", por lo que en un ámbito planetario "la decadencia de un orden político nacional/internacional y el surgimiento de un movimiento social mundial tiene relación de causa-efecto" (Iglesias, 2006:56), de forma que la identificación del precariado con una clase social, implicaría necesariamente una idea de proyecto común al cual no se adhiere; por el contrario, sus carencias materiales representadas por su propia situación de inseguridad, imposibilitan cualquier reacción coordinada, dejando a los trabajadores que se encuentran en estas condiciones a merced del sistema, ya sea por su explotación o por su supresión involuntaria como fuerza de trabajo.

Este planteamiento se explica desde la posición defendida por Santiago Armesilla, a partir de la lectura crítica del texto “La dominación británica de la India”, escrito por Karl Marx en 1853, argumentando como el declive de la economía india no se debió exclusivamente por la instauración del capitalismo británico, sino por su tecnología de vapor y mecánica, ante las cuales eran incapaces de competir las técnicas tradicionales, provocando con ello "que muchos trabajadores y artesanos tradicionales emigraran de sus tradicionales lugares de residencia, o que murieran abocados a la mendicidad al haberse quedado sin trabajo” (Armesilla, 2017:115). 
Más de siglo y medio después, para paliar estos efectos se alienta la responsabilidad social corporativa, un término sobre el que no existe consenso respecto a su descripción, al que se incorporan aspectos sociales en los que incide la mala actuación empresarial (como son los abusos de los derechos humanos y la degradación ambiental), pero sin que representen un resultado final en su negocio (por lo que podría interpretarse como planes de caridad o filantrópicos), buscando una mejora de las relaciones de la empresa con la sociedad (Adeyeye, 2012:7-8); mientras que en la realidad el hecho de que el mercado funcione correctamente, se debe precisamente a la posibilidad de dejar desatendidos a los débiles y pobres (Bridsall, 2006:432). Condición que como alerta Standing (2008:16), ante una situación de inseguridad económica personal, no debe esperarse un comportamiento socialmente responsable, por lo que las personas que se encuentren en estas circunstancias pueden reaccionar de formas adversas.

Es lo ocurrido con los movimientos de protesta espontáneos, inadecuados para la obtención de cambios en el sistema, en tanto no converjan en "algo parecido a un partido", un elemento de guía que permita la identificación y satisfacción de lo que realmente se quiere cambiar (Žižek, 2013b:189). En una sociedad como la china, que carece de una garantía legal para la libertad de expresión, la propensión a la protesta muestra el nivel de aceptación a admitirla como una forma legítima de actividad social ( $\mathrm{Su}$, Feng, 2013:47). A pesar de la ausencia de movimientos organizados, con unidad de discurso en la escena sociopolítica, es habitual que los movimientos de protesta empleen Internet como medio para dar publicidad a los abusos cometidos por el sistema, atrayendo así la atención de abogados, medios de comunicación y profesores universitarios (Béja, 2011:7).

Con ello, el grado de éxito dependerá, en opinión de Yang Su y Shizheng Feng, de si en la protesta intervienen personas con vínculos con las autoridades, que les consienta escapar de la persecución estatal (Su, Feng, 2013:61), no habiéndose alcanzado avances por la actuación del gobierno, quien ha impedido la unidad y la creación de alianzas entre las protestas, cuyo único punto débil se presenta en el aumento de la desigualdad, aspecto que podría llegar a desbordar la capacidad de paralización de cualquier actuación reivindicativa ( $\mathrm{Su}$, Feng, 2013:62); en cuyo caso vendría a confirmar que la dinámica de cambio procedería del conjunto de la clase trabajadora pauperizada, o del liderazgo ofrecido por elementos dirigentes del propio sistema, por lo que al no producirse una ruptura en la dirección, solo se lograrían nuevas refundaciones del mismo.

La propuesta de Standing al considerar a los jóvenes con altos estudios como la fuerza con capacidad de cambio (Standing, 2014b:11), coincidiría con el análisis efectuado por Žižek, al describir la figura de Bill Gates y su percepción por el público mayoritario “como un antiguo hacker que ha 
García., V., B.

triunfado, entendiendo por hacker al subversivo/marginal/anti-elitista que altera el funcionamiento normal de los grandes imperios burocráticos" subyaciendo el mensaje de conversión del "gamberro marginal y subversivo que se adueñó del poder y se presenta ahora como un respetable empresario" (Žižek, 2008:87); que podríamos hacer extensible a otras figuras como Jeff Bezos y Steve Jobs, fundadores de Amazon y Apple, respectivamente. Desde la perspectiva de la hegemonía de Gramsci:

"en el sistema hegemónico existe democracia entre el grupo dirigente y los grupos dirigidos en la medida en que el desarrollo de la economía y por consiguiente de la legislación, que expresa tal desarrollo, favorece el pasaje (molecular) de los grupos dirigidos al grupo dirigente” (Gramsci, 1984:193).

Podríamos encontrar un diálogo entre el planteamiento gramsciano y el definido por Laclau, atendiendo a la explicación de Žižek, a partir de la cual la sociedad se encuentra suspendida entre dos posiciones coincidentes, “una visión corporativa pura (...) donde cada parte ocupa su lugar correspondiente y la visión revolucionaria radical de antagonismo entre la sociedad y las fuerzas antisociales” (Žižek, 2004:98).

Al identificar la deducible reacción adversa ante privaciones sociales, con los estallidos espontáneos multitudinarios, si las demandas de indignación no son capaces de condensarse en una estructura de partido (por las circunstancias a que nos somete el mercado), la concepción de la alta cualificación como contrafuerza de alteración del orden establecido, no es sino una reinterpretación del american way of life (vida, libertad y búsqueda de la felicidad), desde una perspectiva globalizada y neoliberal. Se entiende que todo es posible en el sistema mundial, y que el individuo capaz tiene la fuerza para cambiar las cosas, siendo él el modelo de revolucionario que produce el neoliberalismo, cuyas habilidades de transformación responden a las formas de producción postindustriales, que superan la tradicional estructura Estado-nación y la dinámica partido-sindicato.

Es la ilusión de aparente cambio que se ofrece desde el ecologismo, descrito por Martha E. Gimenez, como "un término genérico y multifacético que se aplica a una serie de ideologías heterogéneas, cuyas perspectivas políticas, teóricas y prácticas, se trazan en la relación de las poblaciones humanas con la naturaleza" (Gimenez, 2000:292). Las protestas hoy, identificables desde los lineamientos de la lucha por la protección ambiental y contra el calentamiento global, crea 
movimientos que encubren un aparente cambio para que todo siga igual, en una perversión del gatopardismo.

En China es reconocida la importancia que desempeña en su economía la aparición y crecimiento de la clase media, por su capacidad de consumo y por sus implicaciones ecológicas ante el incremento de hogares que quieren aumentar su gasto (Hefele, Dittirch, 2011:58); por lo que confrontando este fenómeno al precariado, se extrae que no es sino una confusión de clase, donde la capacidad económica excluye la condición social, es decir, la precarización del trabajo genera unas condiciones de vida de clase baja, pero los mensajes dictados por el mercado y la obtención de un mayor consumo, implica una expansión de la mentalidad de clase media/burguesa e individualista, sin proyecto común.

Para que se den posibilidades de un cambio real, debe generarse conciencia de la existencia mísera a que es sometido el individuo, empatizando con las idénticas circunstancias que deben experimentar sus congéneres. Pensemos en los problemas medioambientales, que de acuerdo con Sacristán, además de ser “problemas políticos, no ideológicos”, son a su vez "globales, internacionales”, sin que su solución sea posible desde posiciones nacionalistas (Sacristán, 2009:182); una situación que evidencia como el mercado no muestra los verdaderos costes sociales de la contaminación, puesto que las empresas no internalizan los efectos provocados en las comunidades polucionadas, como tampoco lo hacen los países respecto de sus emisiones territoriales de gases de efecto invernadero, que repercuten negativamente a escala mundial (Birdsall, 2006:431).

Sacristán exponía que "un rasgo común a todos los marxismos es la crítica de esta sociedad y el intento de identificar racionalmente los factores y los agentes de una posible sociedad justa y emancipada", espacio en que se enmarca la inquietud ante los problemas medioambientales (Sacristán, 2009:169), junto a los que deben ir asociados los sanitarios, por su intrínseca relación a la degradación de la vida. La importancia de la naturaleza radica en que sus límites materiales lo son también de la organización de las fuerzas y relaciones sociales, y productivas humanas (Gimenez, 2000:301).

Si bien la acumulación de Capital (que requiere de una constante expansión de la división de la naturaleza y el trabajo), rompe con el ciclo natural, los monopolios agrícolas a gran escala se han vuelto hegemónicos por su promoción de la riqueza privada, a expensas de la pública, causando una ruptura en la fisiología de la expansión (Clark, Bellamy Foster, 2010:152-153). 
García., V., B.

Esta coyuntura debe abordarse desde el daño medioambiental provocado por la contaminación. En China a escala nacional, 5 millones y medio de hectáreas de suelo están contaminadas por acción de antiguas industrias pesadas (Li, Jiao, Xiao, Chen, Chang, 2015), denunciándose en 2011 la contaminación con metales pesados de 12 millones de toneladas de arroz (Mahr, 2011), alimento básico del pueblo, que tenía en 2017 un consumo anual de $126 \mathrm{~kg}$ por persona (Helgi Analytics, 2019).

Se ha confirmado en China que el vertido de aguas residuales en áreas densamente pobladas ha contribuido al aumento de la tasa de cáncer, siendo la principal causa de muerte en el país (Ebenstein, 2012:200), habiéndose demostrado que el crecimiento económico de la nación, siempre va aparejado a la producción y a los cambios en la polución industrial urbana (Zheng, 2015:29), sin tener en cuenta el debido cuidado medioambiental y sanitario de la población.

La apertura de China al libre mercado llevó a una precarización de la sanidad, como consecuencia de su privatización, ilustrando la situación el hecho de que en 2002, el 45\% de la población urbana y el $79 \%$ de la población rural no tenían seguro médico, caso evidenciado durante el brote de la enfermedad SARS de 2003, que llegó a ser comparado en la comunidad internacional con el accidente de Chernobyl en la URSS (Li, Chen, Powers, 2012:630-631); y si bien hoy el seguro es obligatorio para los trabajadores de las áreas urbanas (en 2014 había 283 millones asegurados) no así queda cubierto el de sus familiares, siendo opcional para los residentes en las zonas urbanas (dando servicio a 314 millones de trabajadores autónomos, niños, estudiantes y personas de la tercera edad) (Fang, 2016), quedando excluidos de él los migrantes rurales (Milcent, 2010:45).

Desde el sistema actual los problemas medioambientales son planteados con una óptica mercantilizadora, que trata de tasar los recursos naturales como capital natural, y el clima como un mercado de emisiones (Clark, Bellamy Foster, 2010:153), al igual que ocurre con la salud humana, de tal modo que la protección del medioambiente y la sanidad deben centralizarse como elementos catalizadores de la lucha, por el cambio total de las condiciones materiales del precariado.

La máxima ofrecida por Manuel Sacristán, al decir que “los grupos revolucionarios tienen que ser ecologistas, y los ecologistas tienen que ser socialmente revolucionarios", en tanto que "una práctica ecologista choca inmediatamente con el presente modo de producción" (Sacristán, 2009:171), demuestra por qué la teoría y práctica ambientalista debe confrontar los problemas generados por las condiciones materiales existentes en la sociedad, y no al margen de esta para alcanzar el cambio. 
Sirva de ejemplo la explicación de Žižek sobre la unificación de fuerzas antagónicas polacas contra el comunismo soviético, en una alianza forjada desde planteamientos prepolíticos, basado en la mera condición de solidaridad entre seres humanos (Žižek, 2001:191), que retoma a partir de la pandemia mundial de 2020, de la enfermedad infecciosa COVID-19, donde ante una tragedia global que sobrepasa la perspectiva nacional, surge una solidaridad de igual magnitud para encontrar una solución, que lleva a reflexionar sobre las condiciones de nuestro sistema vigente en la búsqueda de un cambio radical, a través de la confianza mutua en las personas y la ciencia (Žižek, 2020).

En opinión de Sacristán, "no hay antagonismo entre tecnología y ecologismo, sino entre tecnologías destructoras de las condiciones de vida de nuestra especie y tecnologías favorables a largo plazo a ésta”, pues “no se trata de adorar ignorantemente una naturaleza supuestamente inmutable y pura, buena en sí, sino de evitar que se vuelva invivible para nuestra especie” (Sacristán, 2009:176), por lo que al no existir contradicción, si se logra conjugar las ideas ambientalistas con la praxis tecnológica, puede conseguirse una refundación de los aparatos productores y reproductores, en la medida en que el precariado sea consciente de su existencia y abandone su estado de ignorancia, en una transición a su consolidación como clase madura y definida.

Las condiciones que existen para el precariado, permiten sobrepasar las dificultades a las que aludía Sacristán, cuando consideraba que los movimientos ecologistas debían "hacer ver a la izquierda obrera que, por causa de los problemas ecológicos, algunos de sus intereses a corto plazo están entrando en conflicto con sus intereses a plazo medio y largo" (Sacristán, 2009:173), hechos a los que habría de sumarse la cuestión sanitaria, en la medida en que la acción de movilización del proletariado ha sido desmantelada y recluida a la defensa de los derechos que aún mantiene, mientras que el precariado, al nacer apartado del Estado de bienestar (y consumo), no cuenta con los limitantes a corto plazo, pues sus metas se encuentran en el medio y largo plazo en forma de alteración de la naturaleza, a fin de obtener las condiciones garantes de dignidad personal y sostenibilidad de su salud, y del medio ambiente.

\section{3.-MÉTODO}

\section{Diseño}

La presente investigación responde a una metodología cualitativa, por medio del análisis de los postulados del precariado expuestos por Standing, contrapuestos a autores marxistas que desarrollaron y defendieron la clase social del proletariado, a través de la interpretación de obras seleccionadas, con el 
García., V., B.

objetivo de negar la existencia del precariado como una nueva clase social emergente distinta de la clase trabajadora, lo que realmente es, pero pauperizada por las condiciones exigidas.

\section{Instrumentos}

Para la construcción del marco teórico-conceptual de la negación del precariado como nueva clase social emergente, se consultó el libro en que Standing definió qué es el precariado, cuatro (4) artículos de su autoría en los que desarrolla su trabajo, confrontándolo a los textos de Antonio Gramsci, Karl Marx, Louis Althusser, y Mao Tse-Tung, como exponentes del marxismo clásico, y Slavoj Žižek, por representar una fuente contemporánea de esta escuela de pensamiento.

\section{Procedimiento}

El sistema empleado ha consistido en la identificación de razonamientos e ideas, procedentes de Antonio Gramsci, Karl Marx, Louis Althusser, Mao Tse-Tung, y Slavoj Žižek, con el objetivo de interpretarlas y contraponerlas a la visión defendida por Standing, desde una posición crítica y justificada, argumentando por medio de publicaciones contemporáneas, en las que se manifiesta la contradicción de las condiciones materiales existentes actualmente en China.

\section{4.- CONCLUSIONES}

Un fantasma recorre el mundo: el fantasma del precariado. Este espectro no es nuevo, como tampoco lo es su constitución como clase original, al carecer de conciencia de su propia existencia ni identificación entre los sujetos a ser considerados sus integrantes, sin que dispongan de un plan de acción común ni unos objetivos delimitados. Es el cúmulo de circunstancias superpuestas por el empeoramiento de las condiciones laborales y la desprotección de los trabajadores, el desarraigo provocado por las migraciones forzosas, y el conformismo ante unas circunstancias que sobrepasan las capacidades de un individuo desorganizado e indefenso ante los efectos de la globalización y la incapacidad de las instituciones nacionales.

Es momento de asumir el fracaso en los planteamientos de defensa de los trabajadores, y dejar de buscar la salvación entre las cenizas de lo que pudo ser y no fue; pero sin negar la importancia que tiene la representación de la masa en el logro de objetivos, y la necesidad de recuperar la fuerza conjunta de partidos y sindicatos como elementos de liderazgo en la promoción del cambio de las circunstancias 
materiales, por la mejora de las condiciones socio-laborales. Una situación que no oculta la estafa piramidal del sistema, residiendo el engaño sobre la explotación de los ejércitos de reserva, compuestos por la fuerza de trabajo escasamente remunerada o sencillamente sin remuneración, a cambio de una supuesta satisfacción personal por mor al trabajo, la auto realización y el pensamiento positivo, que no es sino el retorno de la servidumbre cuando no de la esclavitud, alimentada por el espejismo de la obtención futura de seguridad económica.

El precariado es el sometimiento de la clase trabajadora, por su inducción al error de auto identificarse con las condiciones de vida burguesas, que los llevan a vivir en unas condiciones que no responden a sus necesidades materiales y que solo insuflan ánimo de obtención de complacencia por medios artificiosos. No obstante a su condición actual, el precariado, el estado a que se dirige el conjunto de la clase trabajadora en el presente, es la columna vertebral de la revolución postindustrial en ciernes, ya que este grupo no es sino el proletariado despojado de su identidad, incapaz de reconocerse como tal, falsamente auto convencido de adocenamiento, desubicado por la falta de refundación de un proyecto común y viable al que asirse, y por la carencia de medios con que afrontar el efecto narcótico de la fantasía consumista; que debe encontrar los medios en su falta de unidad, para lograr revertir la situación, por el shock que afronta con la catástrofe medioambiental, sanitaria, y económica, puesta de manifiesto en 2020 (con la pandemia de COVID-19), y converger a un frente común cuya resultante sea cambiar el sistema.

\section{REFERENCIAS}

Acemoglu, D. y Robinson, J. A. (2012). Por qué fracasan los países. Paidós.

Adeyeye, A. O. (2012). Corporate Social Responsability of multinational corporations in developing countries. Cambridge University Press.

Althusser, L. (2018). Ideología y aparatos ideológicos de Estado. Grupo Editorial Tomo.

Ang, I. (2013) No longer Chinese? Residual Chineseness after the Rise of China. En Kuehn, J., Louie, K. y Pomfret, D. M. (Ed.) Diasporic Chineseness after the Rise of China (pp. 17-31). UBC Press.

Armesilla, S. (2017). El marxismo y la cuestión nacional española. El Viejo Topo.

Australian Marxist Review (2014). Chinese ideo-political education at the university level - equipping tomorrow's builders of socialism. Australian Marxist Review, 57. https://www.cpa.org.au/amr/57/amr-57-05-chinese-ideo-political-education.html 
García., V., B.

Banco

Mundial

(2021).

Índice

de

Gini.

https://datos.bancomundial.org/indicador/SI.POV.GINI? contextual=region\&locations $=C N$ -

SE-US-BR-DE-MX\&name_desc=true

Bauman, Z. (2008). Tiempos líquidos. Tusquests Editores.

Bauman, Z. (2012). Vida de consumo. Fondo de Cultura Económica.

Béja, J.P. (2011). The New Working Class Renews the Repertoire of Social Conflict. China Perspectives. http://journals.openedition.org/chinaperspectives/5535

Birdsall, N. (2006). Why it matters who runs the IMF and the World Bank. en Ranis, G. Raymond Vreeland, J. y Kosack, S. (Ed.) Globalization and the Nation State (pp. 429-253). Routledge.

Bjorklund, E. M. (1986). The Danwei: Socio-Spatial Characteristics of Work Units in China's Urban Society. Economic Geography, 62 (1), 19-29.

Chi, X. Bo, A. Liu, T. Zhang, P. y Chi, I. (2018). Effects of Mindfulness-Based Stress Reduction on Depression in Adolescents and Young Adults: A Systematic Review and Meta-Analysis. Frontiers in Psychology, 9. https://www.frontiersin.org/articles/10.3389/fpsyg.2018.01034/full

Clark, B. Bellamy Foster, J. (2010) Marx's ecology in the 21st century. World Review of Political Economy, 1(1), 142-156.

Contreras Natera, M. A. (2015). Crítica a la razón neoliberal. Akal.

Ebenstein, A. (2012). The consequences of industrialization: evidence from water pollution and digestive cancers in China. The Review of Economics and Statistics, 94 (1), 186-201.

Estlund, C. (2017). A New Deal for China's Workers?. Harvard University Press.

Fang, H. (2016). The Chinese Health Care System. The Commonwealth Fund. https://international.commonwealthfund.org/countries/china/

Feng, W. Gu, B. y Cai, Y. (2016). The End of China's One-child policy. Studies in Family Planning, 8386.

Fifield, A. (2019). Beijing's one-child policy is gone. But many Chinese are still reluctant to have more. The Washington Post. https://www.washingtonpost.com/world/asia_pacific/beijings-one-childpolicy-is-gone-but-many-chinese-are-still-reluctant-to-have-more/2019/05/02/c722e568-604f11e9-bf24-db4b9fb62aa2_story.html

Fleischer, F. (2012). La diáspora china: un acercamiento a la migración china en Colombia. Revista de Estudios Sociales, 42, 71-79.

Gimenez, M. E. (2000). Does ecology need Marx? Organization \& Environment, 13 (3), 292-304.

Gramsci, A. (1984). Notas sobre Maquiavelo. Ediciones Nueva Visión SAIC.

228 Revista Política, Globalidad y Ciudadanía | Vol. 7, Núm. 14, junio - diciembre 2021 | ISSN 2395-8448 | http://revpoliticas.uanl.mx/ 
Gramsci, A. (2009). La política y el Estado moderno. Diario Público.

Harnecker, M. (2020). Los conceptos elementales del materialismo histórico. Siglo XXI.

Hefele, P. y Dittirch, A. (2011). China's middle class. Kas International Reports, 12, 57-73.

Helgi Analytics (2019). Rice consumption Per Capita in China. Helgi Library. https://www.helgilibrary.com/indicators/rice-consumption-per-capita/china/

Hirai, T. (2015). Capitalism and globalization. En Hirai, T. (Ed.) Capitalism and the World Economy. Routledge.

Hughes, N. C. (1998). Smashing the Iron Rice Bowl. Foreign Affairs, 77 (4), 67-77.

Iglesias, F. A. (2006). Globalizar la democracia. Ediciones Manantial.

Irwin, D. A. (2015). Free Trade Under Fire. Princeton University Press.

Kwan Chan, C. y Peng, Z. (2011). From Iron Rice Bowl to the World's Biggest Sweatshop: Globalization, Institutional Constraints, and the Rights of Chinese Workers. Social Service Review, 85 (3), 421-445.

Lansdowne, H. y Wu , G. (2009). Introduction. En Lansdowne, H. y Wu, G. (Ed.) Socialist China, Capitalist China (pp. 1-9). Routledge.

Li, L. Chen, Q. y Powers, D. (2012). Chinese Healthcare Reform: A Shift toward Social Development. Modern China, 38 (6), 630-645.

Li, X.N. Jiao, W.T. Xiao, R.B. Chen, W.P. y Chang, A.C. (2015). Soil pollution and site remediation policies in China: a review. Environmental Review, 23 (3).

Li, Y. (2013). A perspective on health care for the elderly who lose their only child in China. Scandinavian Journal of Public Health, 41, 550-552.

Lo, K.C. (2013). Reconfiguring the Chinese Diaspora through the Eyes of Ethnic Minorities. En Kuehn, J. Louie, K. y Pomfret, D. M. (Ed.) Diasporic Chineseness after the Rise of China (pp. 170186), UBC Press.

Marginson, S.; Van Der Wende, M. (2009). The New Global Landscape of Nations and Institutions. Higher Education to 2030, 2, 17-62.

Mahr, K. (2011, febrero). Heavy metal: 12 million tons of chinese rice contaminated. Time.

https://science.time.com/2011/02/23/heavy-metal-millions-of-tons-of-chinese-rice-contaminated/

Marx, K. (1844) "Primer manuscrito", Manuscritos Económicos y filosóficos de 1844. https://www.marxists.org/espanol/m-e/1840s/manuscritos/man1.htm

Marx, K. (1846) "Feuerbach: oposición entre las concepciones materialista e idealista", La ideología alemana. https://www.marxists.org/espanol/m-e/1840s/feuerbach/4.htm 
García., V., B.

Marx, K. (1850) La lucha de clases en Francia de 1848 a 1850. https://www.marxists.org/espanol/me/1850s/francia/francia3.htm\#fn58

Marx, K. (1852) El dieciocho Brumario de Luis Bonaparte. https://www.marxists.org/espanol/me/1850s/brumaire/brum5.htm

Marx, K. (2009). El Capital, Tomo III / Vol. 8. Siglo XXI.

Marx, K. (2017). Escritos sobre materialismo histórico. Alianza Editorial.

Marx, K. (2018). Manifiesto comunista. Colofón S.A.

Meng, X. (2014). China's Labour Market Tensions and Future Urbanisation Challenges. En Song, L. Garnaut, R. y Fang, C. (Ed.) Deeping reform for China's long-term growth and development (pp. 379-405). ANU Press.

Milcent, C. (2010). Healthcare for migrants in urban China: a new frontier. China perspectives, 4, 3346.

Neuman, S. y Schmitz, R. (2018). Despite the end of China's one-child policy, births are still lagging. NPR. https://www.npr.org/2018/07/16/629361870/despite-the-end-of-chinas-one-child-policybirths-are-still-lagging

Ng, S.H.; Warner, M. (2002). From “iron rice bowl” to labor market in two decades: changing workplace relations in the People's Republic of China on the eve of WTO entry. Journal of East Asian Studies, 2 (2), 7-35.

O’Brien, K. y Deng, Y. (2017). Preventing Protest One Person at a Time: Psychological Coercion and Relational Repression in China. China Review, 17(2), 179-201.

Ohashi, H. (2015). A mixed effect of globalization on China's economic growth. En Hirai, T. (Ed.) Capitalism and the World Economy (pp. 234-254). Routledge.

Qiuyan; Q. (2017). Chinas has new standards on Marxist schools for ideological understanding. Global Times. https://www.globaltimes.cn/content/1068750.shtml

Song, Y. (2014). Losing an only child: the one-child policy and elderly care in China. Reproductive Health Matters, 22 (43), 113-124.

Standing, G. (2008). Economic Insecurity and Global Casualization: Threat or Promise? Social Indicators Research, 88 (1), 15-30.

Standing, G. (2011). The Precariat: The New Dangerous Class. Bloomsbury Academic.

Standing, G. (2012) the Precariat: From Denizens to Citizens? Polity, 44 (4), 588-608.

Standing, G. (2014a) Por qué el precariado no es un concepto espurio. Sociología del Trabajo, 82, 7-15.

Standing, G. (2014b). The Precariat. Contexts, 13 (4), 10-12. 
Su, Y.; Feng, S. (2013). Adapt or voice: class,guanxi and protest propensity in China. The Journal of Asian Studies, 72 (1), 45-67.

Swider, S. (2015). Building China: Informal Work and the New Precariat. Cornell University Press.

Taber, Nick (2018). How Xi Jinping is Shaping China's Universities. The Diplomat. https://thediplomat.com/2018/08/how-xi-jinping-is-shaping-chinas-universities/

Tapia, M. Elfström, M. y Roca-Servat, D. (2018). Bridging social movement and industrial relations theory: an analysis of worker organizing campaigns in the United States and China. En Briscoe, F. King, B. y Leitzinger J. (Ed.) Social movements, stakeholders and non-market strategy (pp. 173-206). Emerald Publishing Limited.

The World Bank (2021a). China. https://data.worldbank.org/country/china

The World Bank (2021b). Global Consumption Database. https://datatopics.worldbank.org/consumption/country/China

The Borld Bank (2021c). Unemployment, total. https://data.worldbank.org/indicator/SL.UEM.TOTL.ZS?locations $=C N$

The World Bank (2021d). Population, total - China. https://data.worldbank.org/indicator/SP.POP.TOTL?locations $=C N$

Tse-Tung, M. (2013). Sobre el tratamiento correcto de las contradicciones en el seno del pueblo: 27 de febrero de 1957. En Tse-Tung, M. (Ed.) Sobre la práctica y la contradicción (pp. 187-239). Akal.

Xie, Y. y Wu, X. (2008). Danwei Profitability and Earnings Inequality in Urban China. The China Quarterly, 195, 558-581.

Yang, Y. (2019). Inside China's crackdown on Young Marxists. Financial Times. https://www.ft.com/content/fd087484-2f23-11e9-8744-e7016697f225

Zhao, X. (2019). Migrants and urban wage: Evidence from China's internal migration. China Economic Review. https://www.sciencedirect.com/science/article/pii/S1043951X1930032X

Zheng, H. Huai, W. Huang, L. (2015). Relationship between pollution and economic growth in China: empirical evidence from 111 cities, Journal of Urban and Environmental Engineering, 9 (1), 22-31.

Žižek, S. (2001). El espinoso sujeto. Paidós.

Žižek, S. (2004). ¿Lucha de clases o posmodernismo? ¡Sí, por favor! En Butler, J. Laclau, E. y Žižek, S. (Ed.) Contingencia, hegemonía, universalidad (pp. 95-140). Fondo de Cultura Económica.

Žižek, S. (2008). En defensa de la intolerancia. Sequitur. 
García., V., B.

Žižek, S. (2013a). Mao Tse-tung, el señor marxista del desgobierno. En Tse-Tung, M. (Ed.) Sobre la práctica y la contradicción (pp. 5-46). Akal.

Žižek, S. (2013b). Answers without Questions. En Žižek, S. (Ed.) the Idea of Communism (pp. 177-206). Verso.

Žižek, S. (2020, febrero). Coronavirus is Kill Bill-esque blow to capitalism and could lead to reinvention of communism, Russia Today. https://www.rt.com/op-ed/481831-coronavirus-kill-billcapitalism-communism/ 